\title{
ANTIMYCOBACTERIAL SUSCEPTIBILITY TESTING METHODS FOR NATURAL PRODUCTS RESEARCH
}

\author{
Juan Gabriel Bueno Sánchez ${ }^{*}$, Vladimir V. Kouznetsov \\ ${ }^{1}$ Grupo de Micobacterias, Instituto Nacional de Salud, Bogotá, D.C., Centro Colombiano de Investigación en Tuberculosis, \\ Colombia; ${ }^{2}$ Laboratorio de Química Orgánica y Biomolecular, Escuela de Química, Universidad Industrial de Santander, A.A. \\ 678, Bucaramanga, Colombia.
}

Submitted: August 19, 2008; Returned to authors for corrections: June 15, 2009; Approved: November 07, 2009.

\begin{abstract}
The emergence of multidrug-resistant strains of Mycobacterium tuberculosis underscores the need of continuous developments on new and efficient methods to determine the susceptibility of isolates of $M$. tuberculosis in the search for novel antimicrobial agents. Natural products constitute an important source of new drugs, but design and implementation of antimycobacterial susceptibility testing methods are necessary for evaluate the different extracts and compounds. A number of biological assay methodologies are in current use, ranging from the classical disk diffusion and broth dilution assay format, to radiorespirometric (BACTEC), dye-based, and fluorescent/luminescence reporter assays. This review presents an analysis on the in vitro susceptibility testing methods developed for determinate antitubercular activity in natural products and related compounds (semi-synthetic natural products and natural productsderived compounds) and the criteria to select the adequate method for determination of biological activity of new natural products.
\end{abstract}

Key words: Natural products, M. tuberculosis, Susceptibility testing

\section{INTRODUCTION}

Human tuberculosis (TB) is a contagious-infectious disease mainly caused by $M$. tuberculosis, which is an aerobic pathogenic bacterium that establishes its infection usually in the lungs (25). About one third of the world's population is currently infected with M. tuberculosis; $10 \%$ of those infected will develop clinical disease, particularly those who also have the human immunodeficiency virus (HIV) infection. The infectious killer disease, - TB, - is the leading cause of death worldwide from a single human pathogen (65). The World Health Organization (WHO) estimates that active cases of tuberculosis afflict seven to eight million people annually, and lead up to three million deaths per year (12).

Although there are different regimens for treating tuberculosis in the practice, they all are far from ideal. Because these regimens are extremely difficult to follow it, WHO recommends a program of directly observed treatment (DOTS) (47). Only $21 \%$ of the world's TB patients were treated under DOTS in 1998. There are five reasons usually given for needing new tuberculosis drugs: (1) to improve current treatment by shortening the total duration of treatment and/or by providing for more widely spaced intermittent treatment, (2) to improve the treatment of multi-drug-resistant (MDR-TB)

*Corresponding Author. Mailing address: Grupo de Micobacterias, Instituto Nacional de Salud, Bogotá, D.C., Centro Colombiano de Investigación en Tuberculosis CCITB. Avenida calle 26 No. 51-20 Zona 6 CAN Bogotá, D.C., Colombia.; Tel.: (0571) 2200926 Fax: (0571) 220 0934.; E-mail: jbueno@ ins.gov.co 
and extensively drug-resistant (XDR-TB) strains, (3) to provide for more effective treatment of latent tuberculosis infection (LTBI) in programs that are able to implement this practice, (4) side effects, especially hepatotoxicity, are an issue, which in some cases forces an untimely treatment termination, and (5) Since the 1960s, there have been few developments in available therapies for the treatment of tuberculosis, although the discovery of streptomycin by Selman Waksman was in $1944(7,25,35,47,49)$.

Because natural products are a proven template for the development of new scaffolds of drugs $(9,13,46)$, they have received considerable attention as potential anti-TB agents (52). Antimycobacterial active compounds have been found not only from terrestrial plants, but also from other organisms such as fungi and marine plants and animals (18).

Using preliminary functional assays for naturally occurring pure compounds as well as extracts from higher and lower forms of plants, microorganisms and marine organisms, it has been noted that inhibitory activity against $M$. tuberculosis is widespread in Nature. Many these tested compounds have been provided from investigators interested in phytochemical biodiversity. Usually, the potential pharmaceutical worth of the isolated molecules or have been derived from medicinal plants remains unknown since data to show that these compounds are adversely affecting mycobacterial survival mechanisms in humans (50).

Various reasons have been put forward to explain the success of natural products in drug discovery: their high chemical diversity, the effects of evolutionary pressure to create biologically active molecules, the structural similarity of protein targets across many species, and so on (33).

Given the past contributions of natural products to drug discovery and given the fall in the number of new medicines introduced each year (FDA drug approvals down from $~ 40$ in 1996 to 20 in 2006), it seems surprising that natural productbased drug discovery has fallen so far out of fashion with the pharmaceutical industry. Some of the reasons behind that shift are caused by the difficulties in accessing natural products in ways that are compatible with high throughput screening (33).
For the above reasons, it is important to develop new strategies for evaluation and discovery of antimycobacterial drugs, the aim of the present review is to provide and discuss different in vitro methods for discover antitubercular agents in natural products research, and offer a guide for researchers to select an adequate method for determinate biological activity.

\section{Activity-Bioassay guided}

Most collections of natural products start as extracts of fresh or dried material prepared by using various solvents. The extracts are complex mixtures of perhaps several hundred different compounds. Traditional bioassay guided fractionation techniques are generally regarded as being too slow to fit into the pace of high throughput screening: the assays may only be run for a few months in an intensive screening campaign, and the purification of active compounds may not be possible in that time frame (33).

The typical process of discovering natural-product hits and their progression towards development include several phases, which the natural product is extracted from the source, concentrated, fractionated and purified, yielding essentially a single biologically active compound. Chromatography is one of the most useful means of separating mixtures of compounds, as a technique to both purify the components and identify them. Primary methods of chromatography in isolation and analysis of natural products include the following: thin layer chromatography (TLC), liquid column chromatography (LC), gas chromatography (GC), high-performance liquid chromatography (HPLC), fast protein liquid chromatography (FPLC), immobilized metal-ion affinity chromatography, and antibody affinity chromatography (62).

The bottleneck in natural products chemistry is, generally, separation/purification of target compounds from complex mixtures and their structure elucidation. The most important tools for structure elucidation of natural products are nuclear magnetic resonance (NMR) and mass spectroscopic (MS) techniques. In addition, infrared (IR) and ultraviolet-visible spectrophotometric (UV-Vis) methods are of importance (20).

In response to the competition from synthetic compound 
libraries, the process of natural product screening has been streamlined. Nowadays, the mechanics of extract preparation and bioassay-guided fractionation are increasingly automated with partially or fully purified materials being used upfront to avoid crude extracts altogether (41). The recent development in the hyphenated techniques, which combine separation technologies such as HPLC and solid phase extraction (SPE) with NMR and MS techniques, has had a substantial impact in shortening the time line for dereplication, isolation and structure elucidation of the natural individual compound presented in the crude extracts (41).

The driving force behind much phytochemical research is the discovery of new biologically active compounds for medicinal or agricultural uses. Biological assays, then, must be carried out in order to identify promising plant extracts, to guide the separation and isolation, and to evaluate lead compounds (57).

The emergence of pathogenic microbes with increased resistance to established antibiotics provides a major incentive for the discovery of new antimicrobial agents. Antimicrobial screening for the phytochemicals from plant extracts, then, represents a starting point for antimicrobial drug discovery (57), especially, antimycobacterial drugs.

\section{Anti-TB in vitro bioassays}

Agar diffusion: The paper disk diffusion bioassay as well as agar diffusion assays were first developed for bacteria (21, 32). Disk methods comprise the placing of filter paper disks containing test compounds on agar plate surfaces previously inoculated with the test organism. The test molecules or plant extracts then diffuse into the agar and inhibit growth of the test microorganisms (32). After incubation, mean diameters of growth inhibition zones are recorded. Recently, a M. marinum zone of inhibition assay has been developed as a method for screening of antimycobacterial compounds from marine organisms extracts, the use of $M$. marinum, which is a ubiquitous aquatic pathogen, makes of this specie a model for to evaluate antitubercular activity of this kind of natural products (6). Diffusion assays are recommended more for polar rather than non-polar molecules or mixture of compounds such as essential oils (40).

These assays employed in many antimicrobial assays for discovery of natural product leads are not quantitative when used to evaluate extracts or new compounds, but are merely an indication that there is growth inhibition at some unknown concentration along the concentration gradient (52). With mycobacteria, there are additional reasons to avoid diffusion assays. A principal problem is that the mycobacteria cell wall is often more susceptible to less-polar compounds. Non-polar compounds will diffuse more slowly than polar compounds in the aqueous agar medium and, thus, giving the erroneous impression of weak activity (52). This problem is encountered when zones of inhibition are compared for different classes of compounds like essential oil compounds, compounds found in aqueous plant extracts, or standard antibiotics (52). Furthermore, agar-diffusion methods are difficult to run on high capacity screening platforms (19).

Agar dilution: In the 1950s, Canetti et al. described the first Drug Susceptibility Testing (DST) method for $M$. tuberculosis, involving the preparation of a concentration series of drugs against $M$. tuberculosis complex in Lowenstein-Jensen medium, inoculation of the bacterial cultures on the slants, and reading of the inhibition of growth by drugs at different concentrations $(10,60)$. The agar dilution tests permit to determine the MIC, however, none of its worked out modifications was repeatedly used over a longer period of time. Disadvantage is the high need of amounts of test compounds (20 mg/plate to test $1.000 \mathrm{mg} / \mathrm{mL}$ ), which restricted its use to easily available test materials $(10,52)$. In essential oil compounds, a solvent or detergent has to be added into the agar medium for homogenization. Volatilization of test material and activity in the vapor phase of a Petri dish may also occur and play a role in determining MIC by this method, affecting a real antimicrobial activity $(34,52)$. Other dilution methods uses a concentration series of antituberculosis drugs, in 7H10 medium, distributed semi-automatically in 25-well plates (60). The major disadvantage with such assays is the time 
requirement to visibly detect growth of the colonies.

Radiometric: BACTEC $460 \AA$ instrument is a semi-automated radiometric drug susceptibility testing (RAD) method that measures the ${ }^{14} \mathrm{CO}_{2}$ produced by metabolic breakdown of (1${ }^{14} \mathrm{C}$ ) palmitic acid in a liquid Middlebrook 7H12 medium (4, 16, 42). With this method, multiple concentrations can be tested and an MIC calculated (16). However, although results are generally available in 5 days, the tests performed on the BACTEC system are costly and are not suited to the evaluation of large numbers of compounds (15). Other radiometric assay system, which can be employed to screen for inhibitors of mycobacterial growth uses a strain of the rapidly growing saprophyte Mycobacterium aurum is used as the test organism. Inhibition of its growth is highly predictive of activity against M. tuberculosis, which cannot itself be used in screening because of its growth characteristics and highly infectious nature. The viability of $M$. aurum in the presence of a test sample is monitored by measuring the uptake of radiolabelled uracil into the cells. In a microtiter plate format, the screen has the potential for testing several thousand samples per day (15). The major disadvantages of these assays are the cost and the isotope disposal in some countries.

Micro broth dilution: Dilution bioassays have one major advantage over diffusion bioassays, namely the test compound concentration in the medium is defined. Consequently, dilution assays are regarded as the method of choice to compare MIC values $(11,56)$. The advent of microtitre plates has led to significant reductions in test compound concentrations; furthermore, in combination with spectrophotometric or fluorometric plate readers it is possible to get enormously throughput $(52,32)$. Whereas the growth of many mycobacteria can be quantitated by turbidity in a liquid medium, the tendency of mycobacteria to clump makes this a difficult test. However, test samples that are not fully soluble may interfere with turbidity readings, making interpretation difficult, emphasizing the need for a negative control or sterility control, i.e. extract dissolved in blank medium without microorganisms $(52,19)$. An important alternative can be the use of oxidation/reduction indicator dyes such as Alamar Blue (27, 36, 37), 3-(4,5-dimethylthiazol-2-yl)-2,5diphenyltetrazolium bromide (MTT) (1, 22), 2,3,5triphenyltetrazolium chloride (TTC) (44), and 2,3-bis(2methoxy-4-nitro-5-sulfophenyl)-5-[(phenylamino)carbonyl]2H-tetrazoliumhydroxide (XTT) (23), with which, the growth/inhibition can be read visually; and the reduced form of these dyes can also be quantitated colorimetrically by measuring absorbance at $570 \mathrm{~nm}$, or fluorimetrically by exciting at $530 \mathrm{~nm}$ and detecting emission at $590 \mathrm{~nm}$; the latter mode is more sensitive. These more quantitative assays are also capable of detecting partial inhibition, making it ideal for determining the relative activity of fractions using one or two concentrations (52). In general, dilution methods are appropriate for assaying polar and non-polar extracts or compounds to determine MIC and MBC/MFC-values (19). However, in the case of essential oils, it is important to take into account that the evaporation and the absorption of components by plastic plates could affect the antimicrobial activity, as far as, broth dilution assays should be carried out under sealed conditions to prevent loss of evaporation, and glass material should be used to prevent loss by absorption (34).

Flow cytometry: The first experiments, in which flow cytometry was used to study the effects of antimicrobial agents in prokaryotes, were carried out at the beginning of the $1980 \mathrm{~s}$ (58). In the 1990s, there were interesting and important advances in this field from microbiology laboratories, and consequently, the number of scientific articles addressing at the antimicrobial responses of bacteria (including mycobacteria), fungi, and parasites to antimicrobial agents, were considerably increased (2).

Fluorescein diacetate (FDA) (a nonfluorescent diacetyl fluorescein ester that becomes fluorescent upon hydrolysis by cytoplasmic esterases) staining and flow cytometry were used by Norden et al. (48) for the flow cytometry susceptibility testing of $M$. tuberculosis. Pina-Vaz et al. stained $M$. 
tuberculosis in the absence or presence of antimycobacterial drugs with SYTO 16 (a nucleic acid fluorescent stain that only penetrates into cells with severe lesion of the membrane) (54). The time needed to obtain susceptibility results of $M$. tuberculosis using classical methodologies is still too long, and flow cytometry is a promising technique in the setting of the clinical laboratory, giving fast results. However, the higher cost of equipment is a limitation of this methodology.

Reporter gene assays: Genes encoding luciferase enzymes have been cloned from several species of firefly, beetle, crustacean, bacteria and the sea pansy (45). Other fluorescent proteins such as the red fluorescent protein (RFP) and green fluorescent protein (GFP) has been used, following their introduction in mycobacteria on plasmids, permits the rapid determination of bacterial viability by measuring the expression of an introduced fluorescent or luminescent protein $(52,3,17)$. Application of these proteins permits to work in multi-well formats with more convenient high throughput detection. This potentially allows the development of screens to be established that utilizes common equipment and procedures to detect activity at different targets (45). Since reporter gene assays are now just one of many options available, and considering that their use for commercial applications is often limited by patent restrictions, the number of laboratories reported to be using this technology for primary screens of natural products is fairly small (45).

High-performance liquid chromatography mycolic acid analysis: Mycolic acid analysis using HPLC and $p$ bromophenacyl bromide derivatizing reagent for UV detection is a well-established method for identification of mycobacterial strains isolated from clinical specimens $(8,61)$. It was found that a linear relationship between the total area under the mycolic acid (TAMA) chromatographic peaks of a culture of M. tuberculosis and $\log$ CFU per mL, suggesting the possibility of using TAMA as a good estimator of mycobacterial growth and also as a means of susceptibility testing of M. tuberculosis $(28,61)$. Although the reagents and supplies for HPLC are more cheaply compared with those needed for Bactec radiometric method, the cost of this equipment limits a more wide use.

\section{Toxicology assays}

Toxicity is a leading cause of attrition at all stages of the drug development process $(5,39)$. In vitro toxicology assays can be divided on the basis of timing and purpose of the application into prospective assays and retrospective assays (5, 39). Prospective in vitro toxicology assays are those assays that are conducted before the first in vivo toxicology studies, and attempt to predict toxicities that are development-limiting. These include assays for general or cell-type-specific cytotoxicity, genotoxicity, hERG (human ether-a-go-gorelated, also known as $\mathrm{KCNH} 2$ ) channel block, drug-drug interactions and metabolite mediated toxicity. These cytotoxicity assays are often among the earliest toxicity assays to be conducted. In vitro cytotoxicity assays can be valuable for interpreting the results of in vitro safety and efficacy assays. Results for many of the in vitro assays detailed below may be confounded by cytotoxicity; indeed, the Ames assay and micronucleus assay include an assessment of genotoxicity that is critical for the interpretation of the assay results $(5,39)$.

\section{Dormant tubercle bacilli assays}

An important problem in tuberculosis control is the persistence of M. tuberculosis despite chemotherapy (24). A stage of latency in tubercle bacilli has been found as principle cause for most of the problems associated with the disease (64). There is still no specific drug available in the market, which could effectively kill this latent bacillus (38). The obstacle in the development of novel drugs is caused to the lack of a screening system, which can determine inhibitors of latent bacilli of tuberculosis (38). Wayne`s hypoxic model is used for in vitro evaluation of new compounds, but posses low throughput capability $(38,63)$. Using a $M$. tuberculosis pFCAluxAB strain, which is $M$. tuberculosis $\mathrm{H} 37 \mathrm{Rv}$ strain containing a plasmid with an acetamidase promoter driving a bacterial luciferase gene, Cho et al. (2007) implemented a 
high-throughput, luminescence-based low-oxygen-recovery assay for screening of compounds against nonreplicating $M$. tuberculosis (14). Recently, Khan and Sarkar (2008) have developed a dormant stage specific antitubercular screening protocol in microplate format using Wayne's hypoxic model and nitrate reductase activity in $M$. bovis BCG (Bacillus Calmette-Guérin) culture (38).

\section{Macrophages assays}

In vitro models of macrophage infection by Mycobacterium spp have been used to assay virulence and the intracellular activity of antimycobacterials (43). A source of macrophages can vary species, including humans, mice and rabbits. The strain of $M$. tuberculosis, used to infect the macrophages, is another source of variability, e.g., $M$. tuberculosis H37Ra, H37Rv, Erdman, and clinical isolates (43). Species of mycobacteria other than M. tuberculosis have also been tested, e.g., M. bovis BCG and M. avium. The species M. tuberculosis is cytotoxic for macrophages are known; the extent of the toxicity depends on all of the variables cited above. The results of macrophage cytotoxicity are most heavily infected die rapidly and become no adherent (43). The activity of selected compounds against intracellular M. tuberculosis can be determined using the murine macrophage cell line RAW 264.7 (ATCC TIB-71) infected with M. tuberculosis luciferase reporter strain pSMT1 (55). Measurement of luminescence has shown to provide a rapid alternative to the counting of colonies as a means of evaluate mycobacterial viability (55).

\section{Criteria for activity}

In anti-infective in vitro models, the activity of extracts or compounds is generally expressed by numeric values $\left(\mathrm{IC}_{50}\right.$, $\mathrm{IC}_{90}, \mathrm{MIC}$, etc.). For a correct interpretation of these efficacy variables, a profound knowledge of the model and the used protocol are required (19). Relevant activity relates to $\mathrm{IC}_{50}$ values below $100 \mu \mathrm{g} / \mathrm{mL}$ for plant extracts $(128 \mu \mathrm{g} / \mathrm{mL}$ if is used the Clinical and Laboratory Standards Institute MIC range), $0.25 \% \mathrm{v} / \mathrm{v}$ for essential oils and below $25 \mu \mathrm{M}$ for pure compounds (19, 29, 31, 53). For whole-cell bacteria activity an optimal value being $\leq 1 \mu \mathrm{g} / \mathrm{mL}$ is required (26). Tosun et al. proposed to consider promising activity in a concentration of $200 \mu \mathrm{g} / \mathrm{mL}$, but the literature reports that "antibacterial" compounds with MICs values greater than $100 \mu \mathrm{g} / \mathrm{mL}$, which are poorly active and their clinical perspective has little relevance in reality an MIC of less than $10 \mu \mathrm{g} / \mathrm{mL}$, and ideally less than $2 \mu \mathrm{g} / \mathrm{mL}$ with a selectivity index $\left(\mathrm{SI}=\mathrm{IC}_{50}\right.$ Vero cells/MIC) of $>10$ is considered as being of interest to pharmaceutical industry $(30,51,59)$.

\section{Perspectives}

Natural products have and will continue to be a major resource for therapeutic products. Until now days, researchers consider them a source of diverse chemical structure that are virtually impossible to replicate in a synthetic chemistry laboratory. The above techniques have been refined into high throughput methods by the application of parallel processing procedures to the chromatographic and analytical steps. Following screening, active fractions are rapidly purified and the structure of the components determined using a rapid and sensitive combination of NMR and mass spectrometry with as little as $50 \mu \mathrm{g}$ of sample. The sequence of procedures developed by this group will undoubtedly contribute to the supply of libraries for the already well-developed highthroughput screening programs. The continuous development of sensitive, rapid and inexpensive assay materials and detection equipment will ensure that researchers in all institutions are able to contribute to the development of our understanding and utilization of natural product resources.

\section{REFERENCES}

1. Abate, G.; Mshana, R.N.; Miorner, H. (1998). Evaluation of a colorimetric assay based on 3-(4,5-dimethylthiazol-2-yl)-2,5-diphenyl tetrazolium bromide (MTT) for rapid detection of rifampicin resistance in Mycobacterium tuberculosis. Int. J. Tuberc. Lung. Dis. 2(12), 1011-1016.

2. Alvarez-Barrientos, A.; Arroyo, J.; Canton, R.; Nombela, C.; SanchezPerez, M. (2000). Applications of flow cytometry to clinical microbiology. Clin. Microbiol. Rev. 13(2), 167-195.

3. Arain, T.M.; Resconi, A.E.; Singh, D.C.; Stover, C.K. (1996). Reporter gene technology to assess activity of antimycobacterial agents in macrophages. Antimicrob. Agents. Chemother. 40(6), 1542-1544. 
4. Ashtekar, D.R.; Fernandes, F.; Khadse, B.G.; Shirodkar, M.V. (1987). A rapid method for the evaluation of new antituberculous agents. Chemotherapy. 33(1), 22-27.

5. Barile, F.A. (2008). Principles of toxicology testing. CRC Press, Boca Raton, USA.

6. Barker, L.P.; Lien, B.A.; Brun, O.S.; Schaak, D.D.; McDonough, K.A.; Chang, L.C. (2007). A M. marinum zone of inhibition assay as a method for screening potential antimycobacterial compounds from marine extracts. Planta. Med. 73(6), 559-563.

7. Barry, P.J.; O'Connor, T.M. (2007). Novel agents in the management of M. tuberculosis disease. Curr. Med. Chem. 14(18), 2000-2008.

8. Butler, W.R., Guthertz, L.S. (2001). Mycolic acid analysis by highperformance liquid chromatography for identification of Mycobacterium species. Clin. Microbiol. Rev. 14(4), 704-726.

9. Butler, M. S. (2005). Natural products to drugs: natural product derived compounds in clinical trials. Nat. Prod. Rep. 22(2), 162-195.

10. Canetti, G.; Froman, S.; Grosset, J.; Hauduroy, P.; Langerova, M.; Mahler, H.T.; Meissner, G.; Mitchinson, D. A.; Sula, L. (1963). Mycobacteria: Laboratory Methods for Testing Drug Sensitivity and Resistance. Bull. World. Health. Organ. 29, 565-578.

11. Chand, S.; Lusunzi, I.; Veal, D.A; Williams, L.R.; Karuso, P. (1994). Rapid screening of the antimicrobial activity of extracts and natural products. J. Antibiot. (Tokyo). 47(11), 1295-1304.

12. Chang-Blanc, D.; Nunn, P. (1999). Incentives and Disincentives for New Anti-Tuberculosis Drug Development. World Health Organization, Geneva, Switzerland.

13. Chin, Y.W.; Balunas, M.J.; Chai, H.B.; Kinghorn, A.D. (2006). Drug discovery from natural sources. AAPS. J. 8(2), E239-E253.

14. Cho, S.H.; Warit, S.; Wan, B.; Hwang, C.H.; Pauli, G.F.; Franzblau, S.G. (2007). Low-oxygen-recovery assay for high-throughput screening of compounds against nonreplicating M. tuberculosis. Antimicrob. Agents. Chemother. 51(4), 1380-1385.

15. Chung, G.A.; Aktar, Z.; Jackson, S.; Duncan, K. (1995). High-throughput screen for detecting antimycobacterial agents. Antimicrob. Agents. Chemother. 39(10), 2235-2238.

16. Collins, L.; Franzblau, S.G. (1997). Microplate alamar blue assay versus BACTEC 460 system for high-throughput screening of compounds against M. tuberculosis and M. avium. Antimicrob. Agents. Chemother. 41(5), 1004-1009.

17. Collins, L.A.; Torrero, M.N.; Franzblau, S.G. (1998). Green fluorescent protein reporter microplate assay for high-throughput screening of compounds against M. tuberculosis. Antimicrob. Agents. Chemother. 42(2), 344-347.

18. Copp, B.R.; Pearce, A.N. (2007). Natural product growth inhibitors of $M$. tuberculosis. Nat. Prod. Rep. 24(2), 278-297.

19. Cos, P.; Vlietinck, A.J.; Berghe, D.V.; Maes, L. (2006). Anti-infective potential of natural products: how to develop a stronger in vitro 'proof-ofconcept'. J. Ethnopharmacol. 106(3), 290-302.
20. Cseke, L.; Setzer, W.; Vogler, B.; Kirakosyan, A.; Kaufman, B. (2006). Traditional, Analytical, and Preparative Separations of Natural Products. In: Cseke, L.; Kirakosyan, A.; Kaufman, B.; Warber, S.; Duke, J.; Brielmann, H.(eds). Natural Products from Plants. CRC Press, Boca Raton, USA, p. 264-317.

21. De Beer, E.J.; Sherwood, M.B. (1945). The Paper-Disc Agar-Plate Method for the Assay of Antibiotic Substances. J. Bacteriol. 50(4), 459467.

22. De-Logu, A.; Uda, P.; Pellerano, M.L.; Pusceddu, M.C.; Saddi, B.; Schivo, M.L. (2001). Comparison of two rapid colorimetric methods for determining resistance of $M$. tuberculosis to rifampin, isoniazid, and streptomycin in liquid medium. Eur. J. Clin. Microbiol. Infect. Dis. 20(1), 33-39.

23. De-Logu, A; Pellerano, M.L.; Sanna, A.; Pusceddu, M.C.; Uda, P.; Saddi, B. (2003).Comparison of the susceptibility testing of clinical isolates of $M$. tuberculosis by the XTT colorimetric method and the NCCLS standards method. Int. J. Antimicrob. Agents. 21(3), 244-250.

24. Dick, T. (2001). Dormant tubercle bacilli: the key to more effective TB chemotherapy? J. Antimicrob. Chemother. 47(1),117-118.

25. Ducati, R.G.; Ruffino-Netto, A.; Basso, L.A.; Santos, D.S. (2006). The resumption of consumption-- a review on tuberculosis. Mem. Inst. Oswaldo. Cruz. 101(7), 697-714.

26. Enna, S.J. (2001). Current protocols in pharmacology. Wiley \& Sons, New York, USA.

27. Franzblau, S.G.; Witzig, R.S.; McLaughlin, J.C.; Torres, P.; Madico, G.; Hernandez, A.; Degnan, M.T.; Cook, M.B.; Quenzer, V.K; Ferguson, R.M.; Gilman, R.H. (1998). Rapid, low-technology MIC determination with clinical Mycobacterium tuberculosis isolates by using the microplate Alamar Blue assay. J. Clin. Microbiol. 36(2), 362-366.

28. Garza-Gonzalez, E.; Guerrero-Olazaran, M.; Tijerina-Menchaca, R.; Viader-Salvado, J.M. (1997). Determination of drug susceptibility of $M$. tuberculosis through mycolic acid analysis. J. Clin. Microbiol. 35(5), 1287-1289.

29. Gibbons, S. (2004). Anti-staphylococcal plant natural products. Nat. Prod. Rep. 21(2), 263-277.

30. Gibbons, S. (2008). Phytochemicals for bacterial resistance--strengths, weaknesses and opportunities. Planta. Med. 74(6), 594-602.

31. Gu, J.Q.; Wang, Y.; Franzblau, S.G.; Montenegro, G.; Yang, D.; Timmermann, B.N. (2004). Antitubercular constituents of Valeriana laxiflora. Planta. Med. 70(6), 509-514.

32. Hadacek, F.; Greger, H. (2000). Testing of Antifungal Natural Products: Methodologies, Comparability of Results and Assay Choice. Phytochem. Anal. 11, 137-147.

33. Harvey, A.L. (2007). Natural products as a screening resource. Curr. Opin. Chem. Biol. 11(5), 480-484.

34. Inouye, S.; Tsuruoka, T.; Uchida, K.; Yamaguchi, H. (2001). Effect of sealing and Tween 80 on the antifungal susceptibility testing of essential oils. Microbiol. Immunol. 45(3), 201-208. 
35. Janin, Y.L. (2007). Antituberculosis drugs: ten years of research. Bioorg. Med. Chem. 15(7), 2479-2513.

36. Jimenez-Arellanes, A.; Meckes, M.; Ramirez, R; Torres, J.; LunaHerrera, J. (2003). Activity against multidrug-resistant M. tuberculosis in Mexican plants used to treat respiratory diseases. Phytother. Res. 17(8), 903-908.

37. Jimenez-Arellanes, A.; Meckes, M.; Torres, J.; Luna-Herrera, J. (2007). Antimycobacterial triterpenoids from Lantana hispida (Verbenaceae). J. Ethnopharmacol. 111(2), 202-205.

38. Khan, A.; Sakhar, D. (2008). A simple whole cell based high throughput screening protocol using $M$. bovis BCG for inhibitors against dormant and active tubercle bacilli. J. Microbiol. Methods. 73(1), 62-68.

39. Kramer, J.A.; Sagartz, J.E.; Morris, D.L. (2007). The application of discovery toxicology and pathology towards the design of safer pharmaceutical lead candidates. Nat. Rev. Drug. Discov. 6(8), 636-649.

40. Lahlou, M. (2004). Methods to study the phytochemistry and bioactivity of essential oils. Phytother. Res. 18(6), 435-448.

41. Lam, K.S. (2007). New aspects of natural products in drug discovery. Trends. Microbiol. 15(6), 279-289.

42. Laszlo, A.; Gill, P.; Handzel, V.; Hodgkin, M.M.; Helbecque, D.M. (1983). Conventional and radiometric drug susceptibility testing of $M$. tuberculosis complex. J. Clin. Microbiol. 18(6), 1335-1339.

43. Lukey, P.; Hooker, E. (2001) Macrophage Virulence Assays. In: Parish, T.; Stoker, N. (eds). M. tuberculosis Protocols. Humana Press, New Jersey, USA, p. 271-280.

44. Mohammadzadeh, A.; Farnia, P.; Ghazvini, K.; Behdani, M.; Rashed, T.; Ghanaat, J. (2006). Rapid and low-cost colorimetric method using 2,3,5triphenyltetrazolium chloride for detection of multidrug-resistant $M$. tuberculosis. J. Med. Microbiol. 55(Pt 12), 1657-1659.

45. New, D.C.; Miller-Martini, D.M.; Wong, Y.H. (2003). Reporter gene assays and their applications to bioassays of natural products. Phytother. Res. 17(5), 439-448.

46. Newman, D. J.; Cragg, G. M. (2007). Natural Products as Sources of New Drugs over the Last 25 Years. J. Nat. Prod. 70(3), 461-477.

47. NIAID. (2001). NIAID Global Health Research Plan for HIV/AIDS, Malaria, and Tuberculosis. NIAID, Bethesda, USA.

48. Norden, M.A.; Kurzynski, T.A.; Bownds, S.E.; Callister, S.M.; Schell, R.F. (1995). Rapid susceptibility testing of M. tuberculosis (H37Ra) by flow cytometry. J. Clin. Microbiol. 33(5), 1231-1237.

49. O'Brien, R.J.; Nunn, P.P. (2001). The need for new drugs against tuberculosis. Obstacles, opportunities, and next steps. Am. J. Respir. Crit. Care. Med. 163(5), 1055-1058.

50. Okunade, A.L.; Elvin-Lewis, M.P.; Lewis, W.H. (2004). Natural antimycobacterial metabolites: current status. Phytochemistry. 65(8), 1017-1032.

51. Orme, I.; Tuberculosis Drug Screening Program. (2001). Search for new drugs for treatment of tuberculosis. Antimicrob. Agents. Chemother. 45(7), 1943-1946.
52. Pauli, G.F.; Case, R.J.; Inui, T., Wang, Y.; Cho, S.; Fischer, N.H; Franzblau, S.G. (2005). New perspectives on natural products in TB drug research. Life. Sci. 78(5), 485-494.

53. Pavan, F.R.; Sato, D.N.; Higuchi, C.T.; Santos, A.B.; Vilegas, W.; Leite, C.Q. (2009). In vitro anti-Mycobacterium tuberculosis activity of some Brazilian “Cerrado" plants. Braz. J. Pharmacogn. 19(1B), 204-206.

54. Pina-Vaz, C.; Costa-de-Oliveira, S.; Rodrigues, A.G. (2005). Safe susceptibility testing of $M$. tuberculosis by flow cytometry with the fluorescent nucleic acid stain SYTO 16. J. Med. Microbiol. 54(Pt 1), 7781.

55. Protopopova, M.; Hanrahan, C.; Nikonenko, B.; Samala, R.; Chen, P.; Gearhart, J.; Einck, L.; Nacy, C.A. (2005). Identification of a new antitubercular drug candidate, SQ109, from a combinatorial library of 1,2-ethylenediamines. J. Antimicrob. Chemother. 56(5), 968-974.

56. Rios, J.L.; Recio, M.C.; Villar, A. (1988). Screening methods for natural products with antimicrobial activity: a review of the literature. $J$. Ethnopharmacol. 23(2-3), 127-149.

57. Setzer, W.; Vogler, B. (2006). Bioassays for Activity. In: Cseke, L.; Kirakosyan, A.; Kaufman, B.; Warber, S.; Duke, J.; Brielmann, H.(eds). Natural Products from Plants. CRC Press, Boca Raton, USA, p. 390413.

58. Steen, H.B.; Boye, E.; Skarstad, K.; Bloom, B.; Godal, T.; Mustafa, S. (1982). Applications of flow cytometry on bacteria: cell cycle kinetics, drug effects, and quantitation of antibody binding. Cytometry. 2(4), 249257.

59. Tosun, F.; Kizilay, C.A.; Sener, B.; Vural, M.; Palittapongarnpim, P (2004). Antimycobacterial screening of some Turkish plants. $J$. Ethnopharmacol. 95(2-3), 273-275.

60. Van-Klingeren, B.; Dessens-Kroon, M.; Van-der-Laan, T.; Kremer, K.; Van- Soolingen, D. (2007). Drug susceptibility testing of M. tuberculosis complex by use of a high-throughput, reproducible, absolute concentration method. J. Clin. Microbiol. 45(8), 2662-2668.

61. Viader-Salvado, J.M.; Garza-Gonzalez, E; Valdez-Leal, R.; Del-BosqueMoncayo, M.A.; Tijerina-Menchaca, R.; Guerrero-Olazaran, M. (2001). Mycolic acid index susceptibility method for M. tuberculosis. J. Clin. Microbiol. 39(7), 2642-2645.

62. Vogler, B.; Setzer, W. (2006). Characterization of Natural Products. In: Cseke, L.; Kirakosyan, A.; Kaufman, B.; Warber, S.; Duke, J.; Brielmann, H.(eds). Natural Products from Plants. CRC Press, Boca Raton, USA, p. 320-387.

63. Wayne, L.G.; Hayes, L.G. (1996). An in vitro model for sequential study of shiftdown of $M$. tuberculosis through two stages of nonreplicating persistence. Infect. Immun. 64(6), 2062-2069.

64. Wayne, L.G; Sohaskey, C.D. (2001). Nonreplicating persistence of $M$. tuberculosis. Annu. Rev. Microbiol. 55, 139-163.

65. Zumla, A.; Grange, J. (1998). Tuberculosis. BMJ. 316(7149), 1962-1964. 